\title{
Visually Improved Understanding of Three-Dimensionally Propagating Electromagnetic Fields in Wireless Networks
}

\author{
J. E. Bendz, H. G. Fernandes, M. K. Zuffo \\ Polytechnic School, University of S. Paulo \\ Integrated Systems Lab (LSI) - Electronic Systems Eng. Dept. \\ Av. Prof. Luciano Gualberto Trav. 3, 05508-900, S. Paulo, SP, Brazil \\ ebendz@lsi.usp.br
}

\begin{abstract}
Visualizations are highly valuable in improving the understanding, as well as the analysis of a variety of physical phenomena. Two such applications can be as a pedagogical tool for enhanced perception of complex topics, or as an everyday aid that helps engineers interpret the outcome of simulations. In this paper we try to meet both these objectives and propose a novel approach to threedimensionally visualize time-varying electromagnetic fields and show how this can be applied to improve the understanding of propagating radio waves used in wireless communication networks. To obtain this, we use a numerical version of Maxwell's equations to create snapshots of a propagating electromagnetic field. In this manner we are able to not only, intuitively get a better general understanding of radio waves, but also disclose important phenomena such as diffraction, reflection, attenuation and multipath behavior.
\end{abstract}

\section{Introduction}

The ability for scientists and engineers to visualize physical phenomena is of utmost importance for correct understanding and analysis. Even more, visualizations have been found to be a valuable complementary pedagogical tool in teaching all sorts of topics such as oceanography [10] or chemistry [11], [1]. For instance, by supplying a group of students with a software for visualizing and animating electromagnetic phenomena, significantly higher understanding was achieved as opposed to a reference group that was taught in a traditional lecture format [2].

In the telecoms and datacoms industries, and especially in the area of wireless networks, there is a great demand for simulations in order to optimize performance and anticipate erroneous constructions of the costly networks. Several methods exist for planning a network, ranging from statistical calculations and empirical models [5] to more computationally heavy deterministic models such as ray tracing [4], [9]. With a higher demand for bandwidth, the trend is to create more sophisticated models in order to capture more parameters from the simulations. This design of new tools require good understanding of the physical principles upon which the simulation tools are to be built.

In this paper we demonstrate a novel approach to threedimensional visualization of an electromagnetic wave propagating through a medium. Especially, we are focusing on electromagnetic waves used in networks of the type IEEE 802.11 (WiFi) and the visualization of the interaction between a wave and typical objects that it might interfere with. The mathematical model used is derived directly from Maxwell's equations and thus, automatically models physical effects such as reflection, attenuation and diffraction. Also, our model works in the time domain and can therefore be programmed to return the time-varying volumetric data continuously or at certain time intervals. This lets us produce three-dimensional animations, which intuitively and pedagogically explain many of the phenomena related to propagating electromagnetic fields.

In section 2 the Finite-Difference Time-Domain, FDTD, method used for the computation of the electromagnetic fields is demonstrated. Here it is also described how the volumetric data is extracted from this model, as well as the design of the source that generates the radio waves. Section 3 briefly discusses the Visualization ToolKit, VTK, and its surface rendering filters used for visualizing the data produced by the FDTD algorithm. In section 4 an application specific preprocessing filter with the purpose to generate smooth surfaces will be derived. In section 5 we will demonstrate the general pedagogical impact of our approach to visualize propagating electromagnetic fields. Snapshots from two simulations will be shown. In the first scenario various physical phenomena will be explained and 
observed, and in the second visualization a more complex environment, imitating an indoor wireless network, will be used as an example of a typical area of application.

\section{Finite-Difference Time-Domain method}

The standard Finite-Difference Time-Domain, FDTD, method was first presented by Yee in 1966 [12]. The FDTD method uses centered finite differences to approximate Maxwell's equations in both the time and space domain. The basic idea of FDTD is to first update the time derivative of the electric fields by use of the magnetic field values, and then update the time derivative of the magnetic field values with these new electric field values. This process is then repeated until the electromagnetic wave has been advanced a certain distance, or until steady state is reached.

\subsection{Stability and accuracy}

There are two main concerns with the FDTD method: stability and accuracy [8]. In order to avoid instability and consequently non-physical behavior in the simulations, the time step size, $\Delta t$, between two consecutive field updates of the same kind (e.g. the electric field values), has to be smaller than the Courant limit:

$$
\Delta t<\frac{1}{c_{0} \sqrt{\frac{1}{(\Delta x)^{2}}+\frac{1}{(\Delta y)^{2}}+\frac{1}{(\Delta z)^{2}}}}
$$

where $c_{0}$ is the speed of light in vacuum and $\Delta x, \Delta y, \Delta z$ are the spatial distances between the grid points in the discretized three-dimensional electrical domain.

The second problem concerning the accuracy, is due to the fact that the finite differences that approximate the time and space derivatives are only second order accurate in time and space, e.g. using a twice as large step size, $\Delta x$, increases the non-physical error in the calculations four times.

These two issues are a main concern in threedimensional electrically large domains, since the need for computer memory scales as a power of three with the distance between the grid points and the total runtime is inversely proportional to the time step size, $\Delta t$. One solution to this problem is to use Higher-Order FDTD, HO-FDTD, [3], which requires twice as many calculations but drastically decreases the numerical errors. Therefore, a larger spatial step size between the grid points can be used and consequently, according to Equation (1), a larger time step, $\Delta t$, is allowed, which in turn decreases the overall total runtime.

\subsection{Signal source}

The electromagnetic emitter used in this paper is a point source, emitting a sinusoidal Gaussian pulse which is centered at the frequency $f_{0}$. There are several reasons for choosing a sinusoidal Gaussian pulse as a source for the signal, where the most important reason from a visualization point of view, is that the propagation of a pulse can easily be shown. Another reason is that the Gaussian behavior gives a smooth contribution of the emitted pulse to the FDTD domain, whereas abrupt pulses will introduce nonphysical random values in a vicinity of the source at the time when the source is turned on. Further, by choosing the pulse to be sinusoidal it is possible to center the pulse around any desired frequency. Finally, the bandwidth within the pulse can be determined by the width of the pulse (a small pulse in the time domain gives a large spectral content in the frequency domain). The numerical expression for this pulse is:

$$
\left.E_{z}\right|_{i_{s}} ^{n}=E_{0} e^{-\left[\left(n-n_{0}\right) / n_{w}\right]^{2}} \sin \left[2 \pi f_{0}\left(n-n_{0}\right) \Delta t\right]
$$

where the index $i_{s}$ denotes the placement of the source in the grid, $\mathrm{n}$ is the actual time step, $n_{0}$ is the midpoint of the pulse, or the time when a Gaussian pulse reaches its maximum. However, note that when $n=n_{0}$, the sinusoidal wave is zero, resulting in a total value of zero for the entire expression. The width of the pulse is determined by $n_{w}$, where a higher value results in a wider pulse.

\subsection{Extraction of volumetric data}

To complete one update step in the time domain, the HO-FDTD algorithm basically runs through all grid points in the discretized volume and first updates all the electric field values and then all the magnetic field values. However, there are actually six volumes that are being calculated at all times, one each for the three electric field values, $E_{x}, E_{y}, E_{z}$ and similarly for the magnetic field values, $H_{x}, H_{y}, H_{z}$.

To be able to visualize a propagating electromagnetic field, these six volumes have to be merged into one single volume. This can be done by first calculating the Poynting vector, $\mathbf{P}=\mathbf{E} \times \mathbf{H}\left[W / \mathrm{m}^{2}\right]$ of the electromagnetic field for all grid points and then take the absolute value of these vectors to obtain scalar values that represent the power density. These extracted scalar values will finally serve as the input for the visualization pipeline.

\section{Visualization with VTK}

The Visualization ToolKit, VTK, is a free, open source software, suitable for scientific visualizations [7]. It was 
chosen here because it contains a wide variety of filters to process the volumetric data with, it is reasonably fast and, of course, it is freely available.

\subsection{Surface rendering}

A propagating electromagnetic pulse can be represented by an advancing wave front. To create such wave fronts, the surface rendering algorithm Marching Cubes [6] was used to produce isovalued surfaces from the three-dimensional data.

The basic principle of the Marching Cubes algorithm, is to divide the volume into small cubes, each with the size of the volume grid resolution, march through all cubes and at every point decide if the vertices of the cube are above or below the isovalue. All cubes are then replaced by appropriate polygons, which are finally put together to the estimated surface.

\subsection{Smoothing filters in VTK}

As described earlier, the source that generates the electromagnetic field is a sinusoidal Gaussian pulse. The pulse will contain several cycles of minima's and maxima's, where the number of cycles depends on the length of the pulse. When the Marching Cubes algorithm compares values above and below a certain isovalue, it will find many corresponding values for every oscillating cycle within this pulse. As a result of this, the algorithm will mount these small parts together into fragments of iso-surfaces, which later will be shown to be highly undesirable. The solution to this would be to try to smooth the data before applying the Marching Cubes algorithm. Some smoothing filters are included in VTK, however, they are designed to deal with small changes in amplitudes, not oscillating sinusoidal functions. Therefore, a new application specific smoothing filter had to be constructed.

\section{Smoothing filter}

To obtain a better visualization of the calculated power density, a smoothing filter that approximates an average envelope of the pulse was included as a post-processing step in the HO-FDTD algorithm after the extraction of the power density values. This smoothing filter can be seen as a running average algorithm that basically runs through all points in the volume, and at every grid point it takes the power density values of the six nearest neighbors in all three directions, adds these to three times the value on the central point and finally divides the total sum with nine.

\subsection{General effects of the filter}

In order to verify the effect of this smoothing filter, a cross section of the calculated absolute values of the Poynting vector was taken along the $\mathrm{x}$-direction of a simulated domain, Figure 1. Psm0 represents the unprocessed data

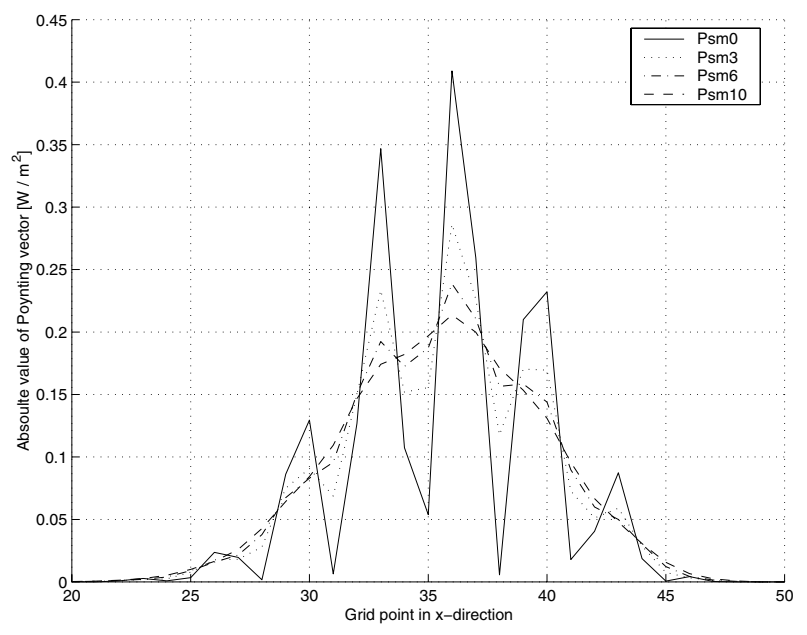

Figure 1. The effect of repeatedly applying the averaging process to the calculated power values before exporting them to the visualization pipeline.

followed by repetitive use of the averaging process 3,6 and 10 times. The effect of this smoothing filter is clearly shown as the minima's are basically canceled out after ten consecutive repetitions. It can also be seen that the overall values in the pulse are averaged and that the final maximum value is equal to half the maximum peak value of the unprocessed data, which is in accordance with theory.

One consequence of the use of this smoothing filter, is that it introduces some broadening of the final pulse due to the averaging procedure that incorporates values of the nearest neighbors. The magnitude and width of this widening can be measured by normalizing the pulses in Figure 1 and then take the logarithmic to be able to estimate the very low power density values at the edge of the pulses. This way the broadening can be approximated to be no more than two grid points at values $40-60 \mathrm{~dB}$ below the maximum value. Thus, this smoothing filter will introduce a slightly broader appearance to the pulse at very low values. However, the visual impact of this is basically insignificant, since during a simulation there is only a need to show values in a range of 30-40 dB at a certain instance. A much larger range than this would introduce too many iso-surfaces with very low values. A much smaller interval is also undesirable since there might be absorbing objects in the simulation setup that can attenuate pulses passing through them with 20-30 dB. 
Therefore it is a requirement to be able to show values of the electromagnetic pulse in a range equal to or larger than this, e.g. isovalues ranging from $0.001 * \mathrm{MAX}$ to $1.0 * \mathrm{MAX}$ $(\Leftrightarrow 30 \mathrm{~dB}$ ), where MAX is the highest value of the power density in the volume at the time when the snapshot is taken.

The result of using the smoothing filter before applying the Marching Cubes algorithm can be seen by observing the rightwards traveling pulse in Figure 2. The upper frame shows the unfiltered volumetric dataset, and it is seen that the Marching Cubes algorithm forms iso-surfaces around all maxima's in the pulse. This clearly complicates the appearance of the propagating electromagnetic wave. A much better representation is observed in the lower frame, where the dataset has been smoothed by running the averaging process ten times.

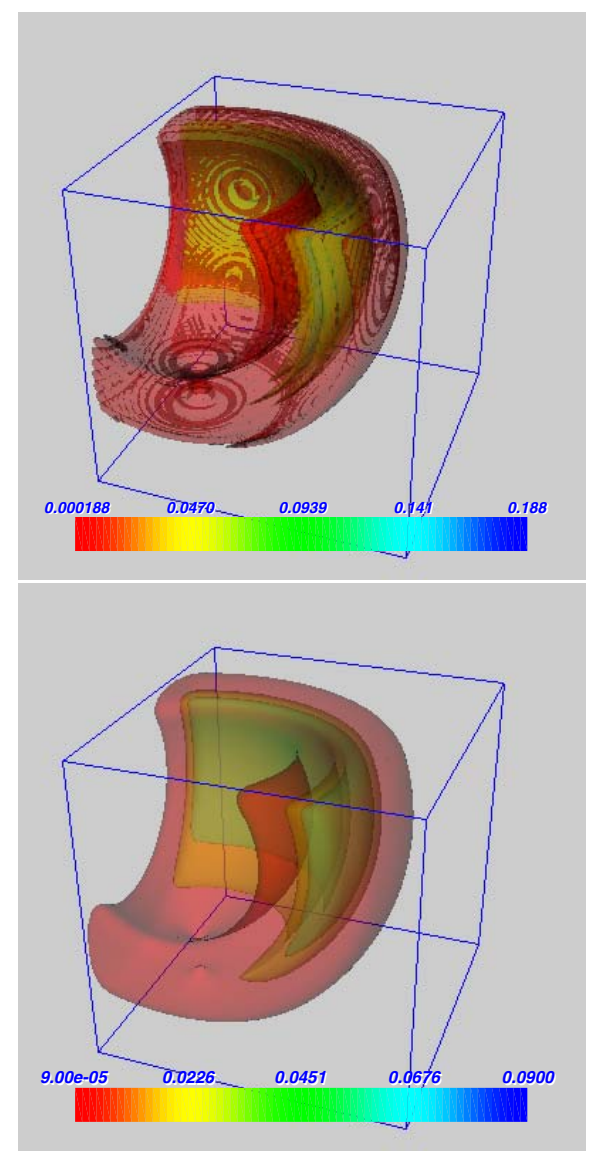

Figure 2. A snapshot of a rightwards propagating pulse in free space. The upper figure shows the unfiltered data. In the lower figure the averaging process has been applied ten times to the volumetric data.

For the Marching Cubes algorithm, minimum and maximum values for the iso-surfaces have to be chosen carefully, as well as the number of iso-surfaces to be shown. The number of iso-surfaces in Figure 2 is set to four. However, the number of scalar values above the highest isovalue are so few that the fourth iso-surface is so small that it can not be seen.

In each frame there is also a color bar included, this is related to the power density of the Poynting vector. The color bars show that the averaging procedure in the smoothing filter decreases the maximum value as expected. The broadening of the pulse can not easily be observed.

Finally, it must be accentuated that it is only the output data to the visualization pipeline that is affected by the smoothing filter. The HO-FDTD simulation continues to run without any kind of smoothing, thus no errors are introduced into the original calculations.

\subsection{Optimizing the filter}

In order to speed up the averaging process of the smoothing filter, as well as the subsequent visualization process, a set of tests was carried out to evaluate the outcome of extracting fewer power values from the HO-FDTD simulations. For instance, by taking only the absolute value of the Poynting vector at every two grid points in the $\mathrm{x}-, \mathrm{y}-$ and $\mathrm{z}$-directions, the amount of volumetric data would decrease to one eight $\left(1 / 2^{3}=1 / 8\right)$ of the original size.

In many ways, this smaller dataset can be beneficiary, for example, less memory is required for a smaller dataset. Also, with fewer data points, the smoothing filter will be much faster, since the averaging process is carried out repeatedly within the filter. Another effect is that with the use of fewer data points, there will also be fewer values between the peaks in a pulse. As a consequence the averaging process can be run fewer times and still it removes the maxima's and minima's.

Table 1 summarizes computational times for three different amounts of extracted grid points. The parameter $q$ represents the rate of decrease of grid points along all three coordinate directions, e.g. $\mathrm{q}=1$ is equivalent to the original case in Figure 2, and $q=2$ means that only every second grid point in all three directions is used. The filter time is the total time for running the averaging process ten times. The average step time is the time for completing a full update cycle of all electric and magnetic fields in the HO-FDTD algorithm, i.e. advancing the pulse one step in time, $\Delta t$. The final column in the table contains a subjective measure of the number of averaging repetitions necessary for erasing the maxima's and minima's. As expected, the processing time of the filter scales well with the number of points in the dataset, e.g. with $\mathrm{q}=1$, the volumetric dataset is $2^{3}=8$ times larger than with $q=2$. It can also be observed that the filtering process for $q=1$ is equally time consuming as a full update step of the HO-FDTD process. This will greatly 


\section{Table 1. Comparison of three different setups of the smoothing filter.}

\begin{tabular}{cccc}
\hline \hline $\mathrm{q}$ & Filter time (s) & Avg. step time (s) & Est. reps. \\
\hline 1 & 0.68 & 0.69 & 8 \\
2 & 0.08 & 0.67 & 6 \\
3 & 0.02 & 0.68 & 6 \\
\hline \hline
\end{tabular}

affect the overall runtime if the smoothing filter is used frequently during the simulation. From the last column it is concluded that fewer averaging cycles are required if there are less points between the peaks in the volumetric dataset. Summarizing the results in Table 1 it is seen that using a value of $q>1$ would be advantageous, especially if the final outcome is an animation which requires a manifold of snapshots and thus a continuously use of the smoothing filter.

However, there is one disadvantage of this optimization of the smoothing filter. In section 4.1 it was seen that the averaging process introduces a broadening of the pulse. Since the filters in this section use a larger and larger physical distance between the points in the data set, it is expected that the broadening of the pulse scales proportionally to q. Measurements were done that confirmed this, i.e. for $q=2$, the pulse broadened four grid points and not two grid points as stated earlier for the case with $q=1$. For $q=3$ the final pulse was 6-7 grid points wider. Thus, it is concluded that $\mathrm{q}=3$ will probably introduce a too large discrepancy from the original pulse shape.

A comparison was finally done in order to visually evaluate the result of the filter optimization, Figure 3. In the left frame the smoothing filter was set to $\mathrm{q}=1$ and the number of averaging repetitions was eight. Corresponding settings for the second frame were $\mathrm{q}=2$ and six repetitions. It is seen

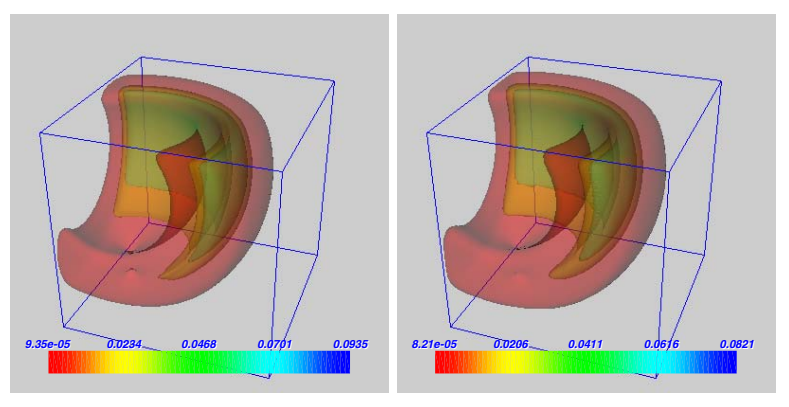

Figure 3. A visual comparison between using $q=1$ (left) and $q=2$ (right) in the smoothing process of the volumetric data. that the two frames are reasonably similar, but a slightly larger broadening of the pulse is implied in the frame to the right. Still, the shorter filter time and the following speedup of the visualization pipeline are two strong reasons for choosing a smoothing filter using $\mathrm{q}=2$.

\section{Results}

Various simulations of propagating electromagnetic waves have been performed in order to visually explain physical phenomena in a pedagogical way, as well as to validate our approach for more complex wireless environments. Primarily we are interested in visualizing electromagnetic waves found in wireless networks of the type IEEE 802.11 (WiFi). For this purpose, a general set of parameters is established for the subsequent simulations.

One of the allowed carrier frequency spectra in the IEEE 802.11 standard is allocated around $2.4 \mathrm{GHz}$, or equivalently $\lambda \approx 0.125 \mathrm{~m}$. The signal source that generates this frequency in our model, is being excited by an electric field in the z-direction, Equation (2), thus little radiation is emitted in the z-direction and an electromagnetic field mainly expanding in the xy-direction and shaped as a donut is expected. Using a uniform grid in all three directions, $\Delta x=\Delta y=\Delta z=\Delta$, and the possibility to use large step sizes with HO-FDTD, the grid point distance is set to: $\Delta=\lambda / 7 \approx 18 \mathrm{~mm}$. The expression for the time step using the HO-FDTD method has to be slightly adjusted from Equation (1) according to [8] and [3], and is here set to: $\Delta t \approx 30 \mathrm{ps}$. This value corresponds to advancing the electromagnetic wave a distance equal to $\Delta t \cdot c_{0} \approx 9 \mathrm{~mm}$ in vacuum during each time step. Thus, snap shots from the HO-FDTD simulation can be taken every $9 \mathrm{~mm}$, or equivalently, a maximum of more than 100 snap shots might be used to make an animation of a propagating wave traveling one meter. Finally, interfering objects in the form of walls and floors are included, assigned material coefficients (relative permittivity and conductivity) corresponding to concrete $\left(\epsilon_{r}=4.0, \sigma=0.146 \mathrm{~S} / \mathrm{m}\right)$. The thickness of the objects is approximately $200 \mathrm{~mm}$, and the absorption is thought to be in the order of $20-30 \mathrm{~dB}$.

\subsection{Visualizing physical phenomena}

In this first part the simulated environment is made up of a single room with the dimensions $3 \mathrm{~m} \times 3 \mathrm{~m} \times 3 \mathrm{~m}$. The setup was purposely chosen simple in order to more easily grasp the interaction between the emitted electromagnetic wave and its surroundings. The simulation was run for 400 time steps and three snapshots were taken after 80, 160 and 320 time steps, Figure 4, which corresponds to an elapsed time of approximately 2.3, 4.6 and $9.2 \mathrm{~ns}$. The grey objects represent segments of concrete walls. The radiating source, 


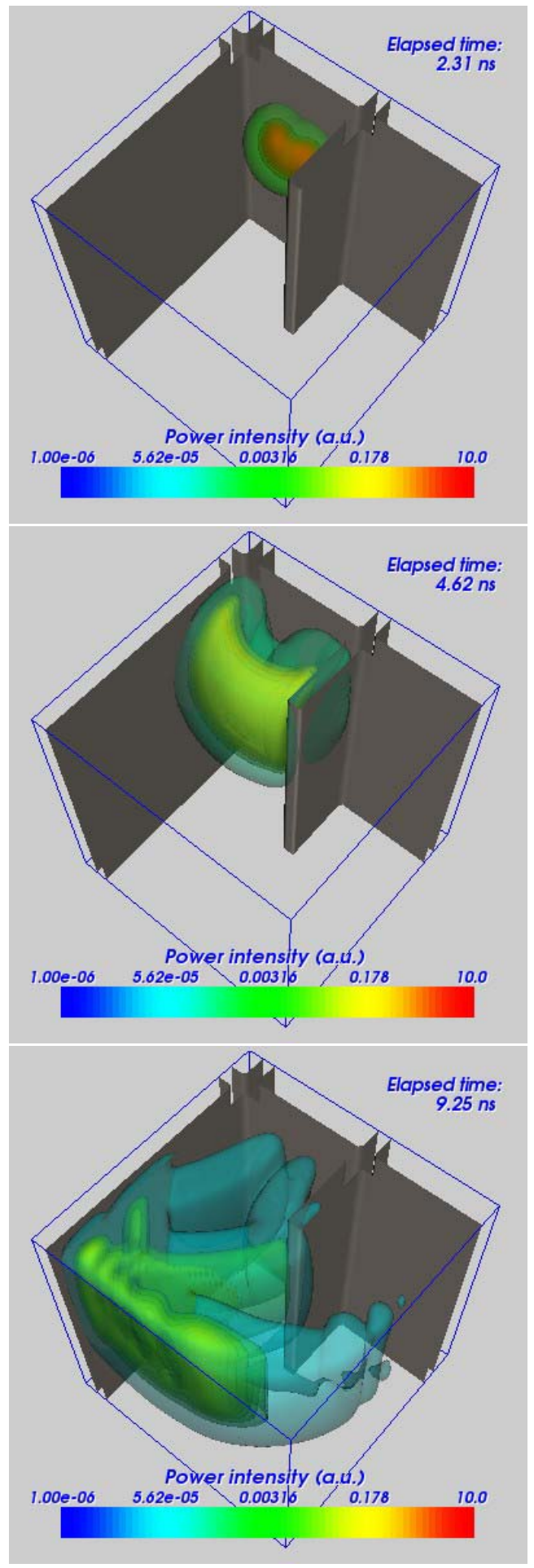

Figure 4. Three snapshots of a propagating pulse. The snapshots were taken after 80 , 160 and 320 time steps. best seen in the first frame, is intended to be installed on one of the walls, at a height of $2.0 \mathrm{~m}$ and approximately $50 \mathrm{~mm}$ in front of it. Because of this arrangement, there will be an immediate reflection in the concrete wall of the backward going part of the pulse that will almost overlap with the forward going part. This is seen as high intensity areas (red) and is an effect of constructive interference between the two parts of the pulse originally traveling in opposite directions.

In the second frame, the effect of absorption can be observed at the wall segment located in the middle. There is a distinct difference in the pulse power intensity in the part of the pulse that is on the left side, as compared to the part of the pulse that has traversed the wall. A very coarse estimation of the absorption can be done by comparing the different colors of the pulse with the incorporated color bar. The result of such a comparison gives an approximate estimation of the absorption in the order of $20 \mathrm{~dB}$. Another physical phenomenon called diffraction is seen at the front edge of the wall segment in the middle, where some part of the pulse has actually been bent around the concrete wall. Further, it is noted that little energy is emitted in the z-direction, as expected since the signal source is applied only to the $E_{z}$ field value and thus the power flow should be directed orthogonally outward from the source direction according to the Poynting vector.

In the third frame several reflections from the concrete walls are clearly visible. These secondary wave fronts are usually referred to as multipath signals and are of a great concern in designing wireless networks. Also, at this point, a continuous decrease of the signal power is observed as the pulse travels further and further away from the source. This can most easily be seen by making an overall comparison of the three frames and observing the absolute value of their corresponding color bars.

\section{$5.2 \quad$ WiFi environment}

For the second simulation a larger and more complex scenario was mounted in order to gather more insight into the complicated behavior of indoor propagating radio waves. Parts of a two-storey building was created by adding walls and floors made of concrete into a $5 \mathrm{~m} \mathrm{x} 4 \mathrm{~m} \mathrm{x} 4.5 \mathrm{~m}$ volume. The reason for choosing two stories was to enhance the usefulness of visualizations of three-dimensional simulations. It is easy to imagine that many of the physical phenomena shown in Figure 4, also would have been visible in a two-dimensional simulation of the xy-plane since the setup used was uniform in the z-direction. On the other hand, for this new scenario seen in Figure 5, we expect to see more variations in the z-direction due to the incorporation of floors / ceilings. To capture information of the radio wave in a reasonably large vicinity of the main wave, isovalues for the creation of iso-surfaces were taken in a $40 \mathrm{~dB}$ 


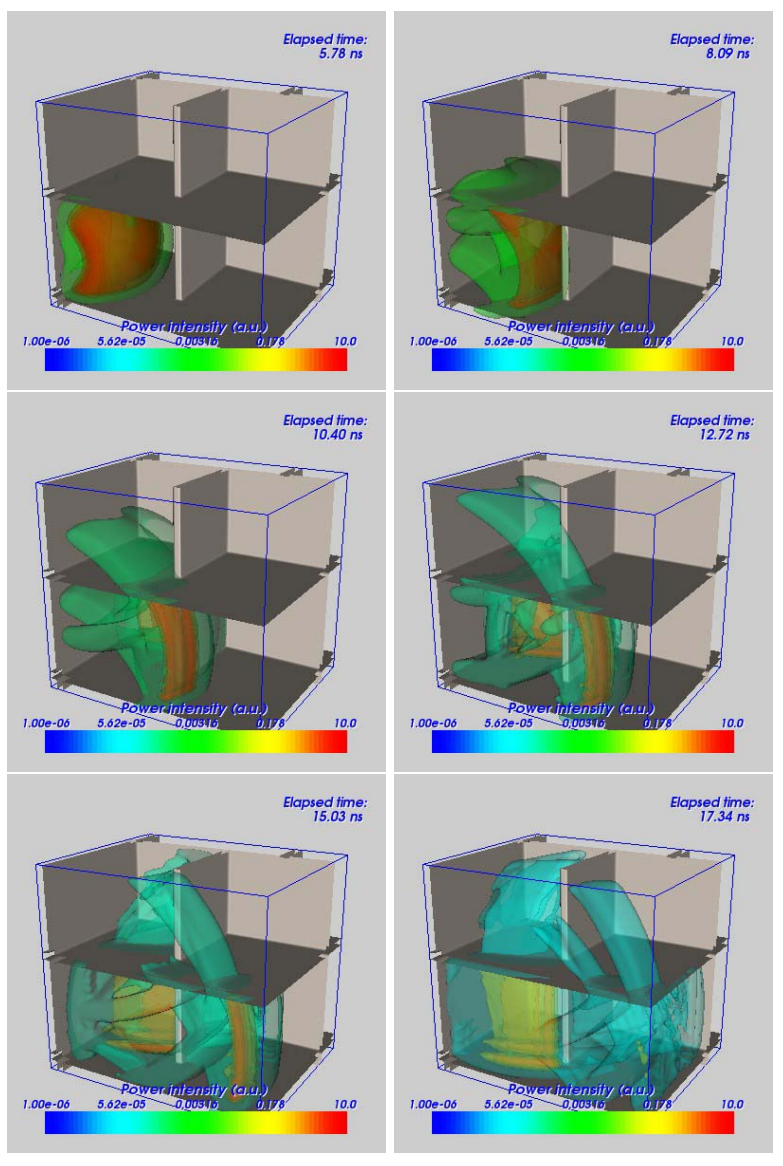

Figure 5. Six snapshots of a propagating pulse. The snapshots were taken after 200 , $280,360,440,520$ and 600 time steps. the second floor. Two remarks can be done for this wave front. First, there is a distinct difference in power density between the two floors due to the absorption in the concrete, and second, there is a small delay between them because the wave front on the upper floor was retarded when it passed through the high permeability concrete layer. As time goes by, more and more reflections are becoming present in the simulation, and adds to the number of copies of the signal in this indoor environment. In the last two frames a reflection from the wall in the background and the wall in the middle is discernible in the left half of the graphs.

This example illustrates well the complicated behavior of decoding the information in a multipath environment. It is clearly seen that there are several copies of the same pulse (information) at many different places and also at the same place but at different times. Looking closer at the last graph in Figure 5, it is seen that the rightwards going pulse on the first floor has just left the simulated domain, whereas a copy of it is still present close to its origin (transmitting antenna), seen as the actual main wave to the left on the first floor. This type of multipath signals reaching a receiving antenna at different times might cause intersymbol interference, ISI, that degrades the performance of the network. For a typical $11 \mathrm{Mbps} 802.11 \mathrm{~b}$ network the time between the data symbols is $1 / 11 \mu s \approx 90 \mathrm{~ns}$. The time delay should typically be less than one third of this value to avoid ISI, the exact value depends on the receiver and how sophisticated it is built. Of course, for even higher transmission bit rates this becomes even more severe, so being able to visualize networks in complex environments is useful to better understand and predict its behavior.

\section{Animations}

To be able to create animations, snapshots of the electromagnetic fields are taken at certain time intervals. Figure 4 showed the behavior of a propagating wave using a relatively long time between the frames. However, with snapshots taken every 10 time steps in the HO-FDTD simulation, frames would be created at a time interval approximately equal to every $0.3 \mathrm{~ns}$, or equivalently after having advanced the pulse $90 \mathrm{~mm}$ in free space. For the purpose of enhancing the static visualizations, such movies were created. The general response to this was a better understanding of the behavior of propagating electromagnetic waves.

\section{Conclusions}

Iso-surfaces have been proved to be one way to successfully visualize electromagnetic wave information contained in a volume. From the results in Figure 2 it was concluded that applying a smoothing filter to the simulated HO-FDTD 
data before exporting the archive to VTK, considerably improved the following visualizations. The effects of reduced maximum values, as well as the broadening of the pulses due to the smoothing filter was also quantified. However, keeping in mind that the final result will be single static figures or animations of a traveling electromagnetic pulse, these unwanted consequences of the smoothing filter will not adversely affect the overall perception.

A series of 3D images, as well as animations, have been produced. These visualizations have been seen to in an intuitively and pedagogically way explain several physical phenomena related to the propagation of radio waves. A complex three-dimensional environment, typically seen in indoor simulations, was finally used to visually improve the understanding of effects that might deteriorate the performance in wireless networks.

\section{References}

[1] D. Ardac and S. Akaygum. Effectiveness of multimediabased instruction that emphasizes molecular representations on students understanding of chemical change. J. of Research in Science Teaching, 41(4):317-337, April 2004.

[2] Y. J. Dori and J. Blecher. Effect of visualizations and active learning on students understanding of electromagnetism concepts. Annual Meeting of the National Association for Research in Science Teaching, NARST, 2003.

[3] J. Fang. Time-Domain Finite Difference Computations for Maxwell's Equations. Ph.D. dissertation EECS Dept., University of California at Berkeley, Berkeley, CA, 1989.

[4] T. Fuegen, J. Maurer, T. Kayser, and W. Wiesbeck. Capability of 3-d ray tracing for defining parameter sets for the specification of future mobile communications systems. IEEE Trans. on Antennas and Propagat., 54(11):3125-3137, November 2006.

[5] M. Jeong and B. Lee. Comparison between path-loss prediction models for wireless telecommunication system design. IEEE Antennas and Propagat. Society International Symposium 2001, 2:186-189, July 2001.

[6] W. E. Lorensen and H. E. Cline. Marching cubes: A high resolution $3 \mathrm{~d}$ surface construction algorithm. ACM SIGGRAPH Computer Grahpics, 21(4):163-167, July 1987.

[7] W. Schroeder, K. Martin, and B. Lorensen. The Visualization Toolkit. Prentice Hall Inc, Upper Saddle River, NJ, 1998.

[8] A. Taflove and S. Hagness. Computational Electrodynamics. The Finite-Difference Time-Domain Method. Artech House Inc, Norwood, MA, 2005.

[9] A. Toscano, F. Bilotti, and L. Vegni. Fast ray-tracing technique for electromagnetic field prediction in mobile communications. IEEE Trans. on Magnetics, 39(3):1238-1241, May 2003.

[10] W. Winn, F. Stahr, C. Sarason, R. Fruland, P. Oppenheimer, and Y. Lee. Learning oceanography from a computer simulation compared with direct experience at sea. J. of Research in Science Teaching, 43(1):25-42, January 2006.
[11] H. K. Wu, J. S. Krajcik, and E. Soloway. Promoting understanding of chemical representations: Students' use of a visualization tool in the classroom. J. of Research in Science Teaching, 38(7):824-842, July 2001.

[12] K. S. Yee. Numerical solution of initial boundary value problems involving maxwells equations in isotropic media. IEEE Trans. on Antennas and Propagat., 14(3):302-307, May 1966. 